\title{
Hepatocellular cancer pain: impact and management challenges
}

This article was published in the following Dove Press journal: Journal of Hepatocellular Carcinoma

\author{
Nathaniel Christian-Miller \\ Catherine Frenette ${ }^{2}$ \\ 'Scripps Clinical Research, Scripps, \\ San Diego, CA, USA; ${ }^{2}$ Scripps Center \\ for Organ and Cell Transplantation, \\ Scripps Green Hospital, La Jolla, CA, \\ USA
}

\begin{abstract}
Hepatocellular carcinoma (HCC) remains the most common primary liver malignancy. Pain comprises one of the most pervasive and troubling symptoms of HCC and may have severely negative effects on patient's quality of life. Furthermore, because HCC frequently arises in the setting of cirrhosis, treating pain related to this malignancy poses a clinical challenge. This article summarizes manifestations of hepatocellular cancer pain, common obstacles to treatment, and practical HCC pain management.
\end{abstract}

Keywords: hepatocellular carcinoma, health-related QOL, analgesic, opioid, loco-regional therapy, cirrhosis

\section{Introduction}

Hepatocellular carcinoma (HCC) has become an increasingly threatening malignancy with regard to both morbidity and mortality. It is responsible for the third greatest number of cancerous deaths worldwide. ${ }^{1}$ Patients who are diagnosed with late-stage $\mathrm{HCC}$, have co-morbid liver diseases, or receive certain loco-regional therapies (LRTs) may suffer great reduction in health-related quality of life (HRQoL). ${ }^{2}$ Interviews with HCC patients revealed fatigue, diarrhea, skin toxicities, and appetite diminishment as key factors affecting the quality of life. Most notably, nine of the 10 patients queried also reported pain, assigning it an important ranking of $\geq 8$ on a $0-10$ scale. Pain has long been a significant concern for HCC patients and their clinicians; it may manifest as abdominal pain, metastatic bone pain, or in some cases, pain related to LRT. ${ }^{3,4}$ More than $80 \%$ of patients who develop HCC have underlying liver disease or cirrhosis, ${ }^{5}$ creating special challenges for pain management. Impaired hepatic function must be taken into account when administering pain medication since most traditional analgesics are metabolized in the liver, and normal doses or dosing frequency can precipitate negative side effects such as hepatic encephalopathy and somnolence. ${ }^{6}$ Procedure-based pain control, especially for patients whose HCC has metastasized beyond the liver, has also been explored. . $^{7,11,22-24}$

In the current review, we attempt to summarize known types of pain associated with HCC, challenges associated with pain treatment, and established and experimental methods for pain treatment. Previous research has demonstrated that HCC patients presenting with pain experience worse outcomes than patients presenting without pain. ${ }^{10} \mathrm{~A}$ more thorough understanding of the pain caused by hepatocellular cancer
Correspondence: Catherine Frenette Scripps Center for Organ and Cell Transplantation, Scripps Green Hospital, 10666 North Torrey Pines Road, \# $200 \mathrm{~N}$, La Jolla, CA 92037, USA

Tel +l 8585544310

Fax + I 8585543009

Email Frenette.Catherine@scrippshealth. org 
and appropriate methods to treat it may thus not only improve patient's HRQoL but also improve patient's prognoses.

\section{Pain classifications}

Right upper quadrant abdominal pain is one of the most commonly reported symptoms for patients with HCC. The pain can be parietal - due to visceral lining inflammation - or visceral. ${ }^{4}$ In either case, abdominal pain is caused principally by visceral encroachment of the primary or metastatic lesion. Recent advances in the understanding of cancer pain increasingly suggest that it may be a distinct entity among other pain states. ${ }^{12}$ It is now thought that the biology of pain precipitated by tumor invasion is defined by cross-talk processes between the immune system and central and peripheral nervous systems and neoplastic cells. However, the relative contribution of this pathophysiology to overall cancer pain is still unknown. Additionally, contemporary data demonstrate that adverse structural and functional changes in the peripheral and central nervous systems may cause patients experiencing chronic abdominal pain to suffer increased pain perception. ${ }^{11}$ Peripheral inflammation and recurrent acute pain contribute to visceral hypersensitivity. At the level of the central nervous system, recurrent acute pain contributes to hyperexcitability in pain-associated brain areas by creating synaptic connections and strengthening existing connections.

Although rarer, $-3-20 \%$ of patients experience HCC that metastasizes to the bone. ${ }^{7}$ These patients often report severe nociceptive pain. Cancer-induced bone pain (CIBP) represents an intricate pain state with nociceptive, inflammatory, and neuropathic characteristics. ${ }^{12}$ Rather than degrading bone directly, cancerous cells provoke osteoclast activation. The acidic environment between osteoclast and bone activates bone sensory neurons via acid-sensing ion channels (ASICs) and transient receptor potential vanilloid receptor 1 (TRPV1), thereby producing bone pain. Cancer cell secretion of chemical mediators (such as prostaglandins and NGF) that can stimulate or sensitize bone nociceptors and compression of sensory nerve fibers by invading tumors also contributes to CIBP.

In an unusual case, a 69-year-old man reporting left pleuritic chest pain was found to have HCC. ${ }^{13}$ Though uncommon, this incidence serves as a reminder that extrahepatic spread of HCC to regions such as the lungs, lymph nodes, skeletal system, and gastrointestinal system may lead to unexpected pain manifestation.

Patients also commonly experience treatment-related pain. Many HCC patients treated with transarterial chemoembolization (TACE) experience postembolization syndrome, characterized by abdominal pain, fever, nausea, and vomiting. ${ }^{3}$ They may also experience pain during the procedure. Benzakoun et $\mathrm{al}^{14}$ found that for patients receiving selective TACE, severe postprocedural pain was associated with an age of $\leq 55$ years, the absence of underlying chronic liver disease, and the administration of a doxorubicin dose of $>50 \%$ of the total theoretical dose during the TACE procedure. For patients whose HCC is in its early stages, radiofrequency ablation (RFA) and microwave ablation are the commonly used LRTs. Postoperative and intraoperative pains are common symptoms of ablative treatments; it has been found that patients who are given general anesthesia as opposed to local anesthesia and who experience more postoperative nausea and vomiting episodes are more susceptible to experiencing intense postoperative pain following RFA. ${ }^{15}$ Furthermore, a positive association has been found between pain intensity and ablation area.

Irreversible electroporation (IRE) is an alternative nonthermal ablation therapy that has generated excitement due to its ability to surmount some challenges of thermal ablation, such as secondary thermal damage. ${ }^{16}$ Informal patient and clinician accounts have also purported that IRE causes less procedurerelated pain than other LRTs. IRE has been shown to be analogous to RFA in terms of both pain experienced and amount of pain medication self-administered for HCC patients. Given its comparable procedure-related pain levels and its ability to bypass some shortcomings of thermal ablation therapy, clinicians may wish to consider IRE among other LRTs for patients who are at increased risk of procedural or postprocedure pain.

The prevalence of pain symptoms in HCC patients has necessitated the development of effective protocols for pain therapy. These therapies are often either drug based or procedure based. While conventional analgesics are used as part of pharmacotherapy, it is important for clinicians to account for impaired hepatic function when administering treatment in order to avoid adverse events related to pain control.

\section{Pain management and associated challenges}

As $\sim 70-90 \%$ of HCC patients have cirrhosis, pain management with traditional analgesics in many patients with HCC presents a clinical challenge. Cirrhosis can alter drug pharmacodynamics by affecting changes in drug absorption, distribution, bioavailability, cytochrome P450 metabolism, and hepatic and renal clearance mechanisms. ${ }^{6}$ Prescription of improper drug classes or failing to closely monitor dosage and dose frequency can cause or worsen variceal hemorrhage, ascites, renal failure, and hepatic encephalopathy or even can precipitate liver failure.

Though nonhepatologists have warily regarded its use in patients with liver disease, acetaminophen is a safe and 
effective pain reliever as long as the patient does not consume alcohol. ${ }^{17}$ The Food and Drug Administration lists $4 \mathrm{~g}$ of acetaminophen as the maximum daily dose, though most hepatologists advise patients consume a maximum of $2 \mathrm{~g} /$ day. Previous survey data suggest a disparity between gastroenterologists and nongastroenterologists regarding overthe-counter analgesic clinician recommendations for cirrhotic patients, ${ }^{6}$ gastroenterologists were more likely to recommend acetaminophen over nonsteroidal anti-inflammatory drugs (NSAIDs), while nongastroenterologists were more likely to recommend the opposite. In practice, NSAIDs are contraindicated for all patients with cirrhosis due to risk for renal failure and gastrointestinal bleeding, even for patients with well-compensated liver disease. ${ }^{17}$ NSAIDs can also precipitate the development of ascites and nephrotoxicity, particularly in patients with portal hypertension.

Opioids are effective for moderate-to-severe pain but should be used cautiously in patients suffering hepatic impairment. Some opioids must undergo biotransformation in the liver to become active metabolites, which can lead to clinical efficacy variability among those with HCC. The reduced analgesic effect exhibited by both tramadol and codeine can be attributed to these phenomena. ${ }^{6}$ Conversely, meperidine bioavailability can increase up to $80 \%$ in cirrhotic patients due to a diminished first-pass effect and can cause CNS suppression and hepatic encephalopathy. ${ }^{18}$ Coupled with the risks posed by its neurotoxic metabolite normeperidine, meperidine should be avoided in all patients with cirrhosis. Clinicians may prefer to use opioids metabolized by glucuronidation, such as morphine and buprenorphine, rather than those metabolized by the cytochrome $\mathrm{P} 450$ system. Liver failure precipitates decreased clearance of opioids metabolized by the P450 system, thereby increasing their bioavailability. Glucuronidation reactions are altered less since glucuronidases are maintained in the setting of hepatic impairment. In light of this, if physicians choose to use medications metabolized via the P450 pathway, dosage should be modified accordingly. Fentanyl, methadone, and hydromorphone are metabolized by the P450 system and may therefore be safer options for cirrhotic patients since they do not produce toxic metabolites. Codeine is also metabolized via the P450 pathway but should be avoided, as its analgesic effects are considerably diminished and accumulation of its metabolite has been known to cause respiratory depression. All opioids should be administered carefully since they can cause or worsen hepatic encephalopathy. The European Association for the Study of the Liver (EASL) notes administering naltrexone along with opioids may help overcome the increased risk of constipation and hepatic encephalopathy in
HCC patients treated with opioids. ${ }^{19}$ A pure opioid receptor antagonist with greater gastrointestinal rather than systemic activity, naltrexone, has been shown to alleviate these risks in populations susceptible to opioid-induced constipation.

For pain management in opioid-naïve cancer patients in general, the National Comprehensive Cancer Network (NCCN) recommends different routes of treatment based on pain intensity. ${ }^{20}$ Patients presenting with severe pain (7-10 on a 1-10 scale) should be given rapid titration of short-acting opioids while those presenting with moderate pain (4-6) should be provided slower titration of short-acting opioids. The NCCN recommends morphine as the starting drug of choice, although other pure agonists such as oxycodone, fentanyl, and hydromorphone are also commonly used and equianalgesic doses may be substituted. In addition to achieve quick analgesic effect, these medications are preferable because their short half-lives allow easier titration than long half-life analgesics such as methadone. Mild cancer pain should be treated first with acetaminophen and adjuvant analgesics such as anticonvulsants and antidepressants. If the pain persists, short-acting opioids may be considered. Anticonvulsants such as gabapentin and pregabalin are commonly used to treat neuropathic cancer-related pain; they may be especially helpful in patients with cirrhosis since these patients frequently experience neuropathies caused by factors such as alcoholism, diabetes, and nutritional deficiencies. ${ }^{20,21}$ Neither gabapentin nor pregabalin is metabolized by the liver, making them the preferred anticonvulsants in the setting of cirrhosis. Antidepressants are also regularly used to treat neuropathic cancer pain. Clinicians wishing to use tricyclic antidepressants (TCAs) should start HCC patients at low doses because of TCAs' sedative effects and because patients may be more affected by anticholinergic adverse effects due to the altered metabolism precipitated by liver disease. Furthermore, clinicians should be vigilant about intestinal stasis as an adverse effect of TCA use since it can cause hepatic encephalopathy. Serotonergic antidepressants should be prescribed carefully since drug interactions pose the risk of serotonin syndrome. Tramadol and tapentadol should not be administered to any patients receiving selective serotonin reuptake inhibitors (SSRIs), serotonin-norepinephrine reuptake inhibitors (SNRIs), or TCAs for this reason.

For pain management in opioid-tolerant patients reporting breakthrough pain $\geq 4$, the NCCN advises administration of a "rescue" dose in addition to the patient's chronic dose. This should be equivalent to $10-20 \%$ of the opioid amount taken by the patient in the preceding 24 hours. Patients should be continually monitored following rescue dose administration to determine efficacy and adverse effects; if the patient's 
pain score remains unchanged, additional rescue doses at $50-100 \%$ the amount of the previous dose may be administered. Adjuvant analgesic therapies may also be considered for opioid-tolerant patients who are only partially responsive to their current medications. Table 1 provides an overview of clinical practice for common analgesics with regard to

Table I NCCN clinical guidelines for various analgesics and suggested modifications in the setting of cirrhosis

\begin{tabular}{|c|c|}
\hline Drug & NCCN clinical guidelines \\
\hline Morphine & $\begin{array}{l}\text { Generally regarded as the standard starting drug of choice in opioid-naïve } \\
\text { patients. Preferably administered orally }\end{array}$ \\
\hline Fentanyl & $\begin{array}{l}\text { Transdermal fentanyl not indicated for rapid titration and should only } \\
\text { be recommended after pain is adequately managed by other opioids in } \\
\text { opioid-tolerant patients. Transdermal fentanyl is effective at reducing } \\
\text { moderate-to-severe cancer pain and may reduce opioid-related } \\
\text { constipation. Sublingual, buccal, and nasal/oral transmucosal formulations } \\
\text { have been shown to effectively manage episodic breakthrough pain }\end{array}$ \\
\hline Hydrocodone & $\begin{array}{l}\text { Should be used as a mild, initial-use opioid, although effective dose } \\
\text { may vary. Only available in immediate-release formulations mixed with } \\
\text { ibuprofen or acetaminophen }\end{array}$ \\
\hline Codeine & $\begin{array}{l}\text { Limited analgesic effect in poor metabolizers and risk of toxicity in rapid } \\
\text { metabolizers. Monitor dosage for safe limits as codeine may only be } \\
\text { available in combined form with acetaminophen or acetylsalicylic acid }\end{array}$ \\
\hline Hydromorphone & $\begin{array}{l}\text { Metabolite may precipitate neurotoxicity. However, has been shown } \\
\text { to reduce pain insufficiently controlled by other analgesics and an RCT } \\
\text { indicated the clinical noninferiority of once-daily hydromorphone relative } \\
\text { to twice daily oxycodone for treating moderate-to-severe cancer pain }\end{array}$ \\
\hline Oxycodone & $\begin{array}{l}\text { Provides similar analgesic and adverse effects to morphine and may be } \\
\text { interchangeable with morphine as initial treatment for cancer pain }\end{array}$ \\
\hline Methadone & $\begin{array}{l}\text { Associated with significantly more drug-drug interactions than other } \\
\text { opioids and difficult to use in cancer patients due to extensive individual } \\
\text { pharmacokinetic variation. Start at doses reduced by } 50 \% \text { of equianalgesic } \\
\text { dose for morphine and gradually increase while providing short-acting } \\
\text { breakthrough analgesics during titration period. Properly titrated } \\
\text { methodone has been shown to be as effective and tolerable as morphine }\end{array}$ \\
\hline
\end{tabular}

Dose adjustment for cirrhotic patients

Reduce dose and dose frequency by $50 \%$

Generally, no adjustment needed for single dose

Active metabolite is hydromorphone. Initial dose should be reduced by $50 \%$

Reduced bioavailability and analgesic effect. Should be avoided

Limited data, although bioavailability and half-life are increased. Dose and dose frequency may need to be reduced

Bioavailability increased 50-95\%. Reduce dose to avoid accumulation

Bioavailability generally unaffected. May require reduced dosing

Bioavailability increased $200-300 \%$. No $>50 \mathrm{mg}$ should be administered every 12 hours and should not be used with SSRIs, TCAs, anticonvulsants, or morphine

Bioavailability increased up to $80 \%$. Avoid using

No adjustment may be necessary, but patient should be closely monitored

Limit dose to $2 \mathrm{~g}$ a day

Limit dose to $300 \mathrm{mg}$ orally a day 900-3600 mg daily in distributed doses 2-3 times per day. Titrate more slowly for the elderly and medically frail. Has been shown to reduce mucositis pain from concomitant radiotherapy and chemotherapy

Pregabalin Starting dose should be $50 \mathrm{mg} 3$ daily and eventually increased to $100 \mathrm{mg}$ 3 times daily. Titrate more slowly for the elderly and medically frail. More efficiently absorbed through Gl tract than gabapentin

Abbreviations: NCCN, National Cancer Comprehensive Care Network; SNRIs, serotonin and norepinephrine reuptake inhibitors; SSRIs, selective serotonin reuptake inhibitors; TCAs, tricyclic antidepressants. 
cancer patients and dosing modifications in the setting of cirrhosis. Given the complexity of treating chronic pain in cancer patients, it would be beneficial to consider consultation with a palliative care specialist to aid in pain management for patients with chronic or difficult-to-control pain.

Pain relief may also be achieved with procedure-based therapy, especially in the setting of metastatic HCC. Radiotherapy has been widely shown to mitigate pain due to bone and lymph node metastases and should be regarded as safe and effective palliation for metastatic HCC symptoms. ${ }^{7,22}$ Current EASL clinical practice guidelines similarly suggest palliative radiotherapy for patients suffering from pain caused by an unambiguous bone metastasis. ${ }^{19}$ However, given its ability to relieve pain more quickly, RFA may be more effective for treating cancer-induced bone pain. ${ }^{23}$ Moreover, Kashima et al achieved pain reduction in $96.6 \%$ of patients with metastatic HCC through RFA compared to studies reporting relief in $50-90 \%$ of patients through radiotherapy. TACE has also been shown to relieve CIBP and may be preferable to radiotherapy since it also achieves palliation more quickly. ${ }^{8}$

Less conventional procedure-based therapies for HCC pain relief have also been explored. Hokotate et $\mathrm{al}^{9}$ successfully treated a Japanese patient's metastatic HCC pain with bone cement therapy, suggesting this as a clinical avenue warranting further research. Electroacupuncture has been shown to alleviate pain in advanced HCC patients as well; however, the onset of significant pain relief was slow, which calls for supplementation with traditional analgesics for this method to be effective. ${ }^{24}$ Transcranial direct current stimulation (tDCS) may prove an effective therapy to alleviate visceral HCC pain. ${ }^{11}$ Multiple sessions of tDCS over the primary motor cortex generated analgesic responses in HCC patients experiencing visceral pain, where relief appeared by the fifth session and persisted for a month. This method may be especially promising because it carries negligible risk and device application is simple. While the analgesic mechanisms of tDCS are not fully understood, resting membrane potential modulation beneath the active electrode and its effects in other parts of the pain-processing network generated by motor cortex-directed inhibition of somatosensation is thought to play a role.

\section{Conclusion}

HCC pain constitutes a serious concern for both patients and providers. It can markedly and negatively impact HRQoL and may result from the spread of HCC itself or LRT. While treating $\mathrm{HCC}$ pain can be clinically challenging due to underlying hepatic impairment, hepatologists have developed analgesic treatment recommendations to bypass the risks of toxic metabolite accumulation and to avoid aggravating hepatic decompensation. Moreover, procedure-based pain therapies such as TACE, radiotherapy, and RFA have been shown to alleviate pain caused by extrahepatic HCC spread. Less studied therapies such as electroacupuncture and tDCS have demonstrated promise and should be explored further. In light of these developments and the disparity between hepatologists and nonhepatologists regarding analgesic preference for cirrhotic patients, coordinated multidisciplinary care between providers may be the optimal route for effective pain management. When used in conjunction with methodical pain assessment, up-to-date clinician knowledge of various forms of HCC pain presentation, and proper analgesic therapy, we hope improved pain management in $\mathrm{HCC}$ patients may be achieved.

\section{Disclosure}

The authors report no conflicts of interest in this work.

\section{References}

1. Waller LP, Deshpande V, Pyrsopoulos N. Hepatocellular carcinoma: a comprehensive review. World J Hepatol. 2015;7(26):2648-2663.

2. Kaiser K, Mallick R, Butt Z, Mulcahy MF, Benson AB, Cella D. Important and relevant symptoms including pain concerns in hepatocellular carcinoma (HCC): a patient interview study. Support Care Cancer. 2014;22(4):919-926.

3. Sun VC, Sarna L. Symptom management in hepatocellular carcinoma. Clin J Oncol Nurs. 2008;12(5):759-766.

4. Kumar M, Panda D. Role of supportive care for terminal stage hepatocellular carcinoma. J Clin Exp Hepatol. 2014;4(suppl 3):S130-S139.

5. Mittal S, El-serag HB. Epidemiology of hepatocellular carcinoma: consider the population. J Clin Gastroenterol. 2013;47(suppl):S2-S6.

6. Lewis JH, Stine JG. Review article: prescribing medications in patients with cirrhosis - a practical guide. Aliment Pharmacol Ther. 2013;37(12):1132-1156.

7. Seong J, Koom WS, Park HC. Radiotherapy for painful bone metastases from hepatocellular carcinoma. Liver Int. 2005;25(2):261-265.

8. Nagata Y, Nakano Y, Abe M, Takahashi M, Kohno S. Osseous metastases from hepatocellular carcinoma: embolization for pain control. Cardiovasc Intervent Radiol. 1989;12(3):149-153.

9. Hokotate H, Baba Y, Churei H, Nakajo M, Ohkubo K, Hamada K. Pain palliation by percutaneous acetabular osteoplasty for metastatic hepatocellular carcinoma. Cardiovasc Intervent Radiol. 2001;24(5):346-348.

10. Carr BI, Pujol L. Pain at presentation and survival in hepatocellular carcinoma. J Pain. 2010;11(10):988-993.

11. Ibrahim NM, Abdelhameed KM, Kamal SMM, Khedr EMH, Kotb HIM. Effect of transcranial direct current stimulation of the motor cortex on visceral pain in patients with hepatocellular carcinoma. Pain Med. 2018;19(3):550-560.

12. Chwistek M. Recent advances in understanding and managing cancer pain. F1000Res. 2017;6:945.

13. Deutsch M, Vasiliou K, Papatheodoridis GV. Hepatocellular carcinoma presenting with pleuritic pain. Eur J Intern Med. 2006;17(3):222.

14. Benzakoun J, Ronot M, Lagadec M, et al. Risks factors for severe pain after selective liver transarterial chemoembolization. Liver Int. 2017;37(4):583-591. 
15. Hsieh YC, Yap YS, Hung CH, Chen CH, Lu SN, Wang JH. Factors related to postoperative pain among patients who underwent radiofrequency ablation of hepatocellular carcinoma. Clin Radiol. 2013;68(6):600-607.

16. Narayanan G, Froud T, Lo K, Barbery KJ, Perez-rojas E, Yrizarry J. Pain analysis in patients with hepatocellular carcinoma: irreversible electroporation versus radiofrequency ablation-initial observations. Cardiovasc Intervent Radiol. 2013;36(1):176-182.

17. Ge PS, Runyon BA. Treatment of patients with cirrhosis. NEngl JMed. 2016;375(21):2104-2105.

18. Soleimanpour H, Safari S, Shahsavari Nia K, Sanaie S, Alavian SM. Opioid drugs in patients with liver disease: a systematic review. Hepat Mon. 2016;16(4):e32636.

19. European Association for the Study of the Liver. EASL Clinical Practice Guidelines: management of hepatocellular carcinoma. J Hepatol. Epub 2018 Apr 5:doi: 10.1016/j.jhep.2018.03.019.
20. National Comprehensive Cancer Network. Adult Cancer Pain (2.2017). 2018. Available from: http://www.nccn.org/professionals/physician_gls/ pdf/pain.pdf. Accessed January 15, 2018.

21. Chandok N, Watt KD. Pain management in the cirrhotic patient: the clinical challenge. Mayo Clin Proc. 2010;85(5):451-458.

22. Kalogeridi MA, Zygogianni A, Kyrgias G, et al. Role of radiotherapy in the management of hepatocellular carcinoma: a systematic review. World J Hepatol. 2015;7(1):101-112.

23. Kashima M, Yamakado K, Takaki H, et al. Radiofrequency ablation for the treatment of bone metastases from hepatocellular carcinoma. AJR Am J Roentgenol. 2010;194(2):536-541.

24. $\mathrm{Xu} \mathrm{L,} \mathrm{Wan} \mathrm{Y,} \mathrm{Huang} \mathrm{J,} \mathrm{Xu} \mathrm{F.} \mathrm{Clinical} \mathrm{analysis} \mathrm{of} \mathrm{electroacupunc-}$ ture and multiple acupoint stimulation in relieving cancer pain in patients with advanced hepatocellular carcinoma. J Cancer Res Ther. 2018;14(1):99-102.
Journal of Hepatocellular Carcinoma

\section{Publish your work in this journal}

The Journal of Hepatocellular Carcinoma is an international, peerreviewed, open access journal that offers a platform for the dissemination and study of clinical, translational and basic research findings in this rapidly developing field. Development in areas including, but not limited to, epidemiology, vaccination, hepatitis therapy, pathology and

Submit your manuscript here: https://www.dovepress.com/journal-of-hepatocellular-carcinoma-journal

molecular tumor classification and prognostication are all considered for publication. The manuscript management system is completely online and includes a very quick and fair peer-review system, which is all easy to use. Visit http://www.dovepress.com/testimonials.php to read real quotes from published authors. 